\title{
COMPLETELY FAITHFUL MODULES AND SELF-INJECTIVE RINGS
}

\author{
GORO AZUMAYA
}

Dedicated to the memory of Professor TADasi Nakayama

A left module over a ring $A$ is called completely faithful if $\Lambda$ is a sum of those left ideals which are homomorphic images of $M$. The notion was first introduced by Morita $[9]^{11}$, and he proved, among others, the following theorem which plays a basic role in his theory of category-isomorphisms: if a $\Lambda$-module $M$ is completely faithful, then $M$ is finitely generated and projective with respect to the endomorphism ring $\Gamma$ of $M$ and $\Lambda$ coincides with the endomorphism ring of $\Gamma$-module $M . \quad \S 1$ of the present paper is devoted to summerize, with some supplements and refinements, Morita's theorems centering around the notion of completely faithful modules. Now, the above theorem implies in particular every completely faithful modules is faithful. However the converse is not necessarily true, so that there naturally arises a problem to find out possible types of ring $\Lambda$ for which every faithful module is completely faithful. In $\S 2$ we shall give a complete answer to the problem: in order that every faithful left module be completely faithful it is necessary and sufficient that $\Lambda$ be left self-injective and a direct sum of indecomposable left ideals having minimal left subideals. The rings characterized here may be regarded as a natural extension of quasi-Frobenius rings which have been largely studied by Nakayama $[11,12] .^{2)}$ In fact, the theorem of Nesbitt and Thrall [14] that every faithful representation of a quasi-Frobenius algebra contain the reduced regular representation as a direct constituent means actually that quasi-Frobenius algebras provide a typical example for the problem.

1. Let $\Lambda$ be a ring with identity element 1 . Let $M={ }_{\Delta} M$ be a (unital) left $\Lambda$-module. If we let $f$ range over all $\Lambda$-homomorphisms of $M$ into $\Lambda$, the

Received September 3, 1965.

1) Cf. also Appendix of Auslander-Goldman [1].

2) Cf. also Eilenberg-Nakayama [6] and Ikeda [7]. 
sum of all homomorphic images $f(M)$ of $M$ is (not only a left but also) a twosided ideal of $\Lambda$, because for any element $\lambda$ of $\Lambda f(M) \lambda$ is the homomorphic image of $M$ by the $\Lambda$-homomorphism $f \lambda: x \rightarrow f(x) \lambda, x \in M$; the two-sided ideal is called the trace ideal of ${ }_{\Lambda} M$. We shall call $M$ a completely faithful ${ }^{3)}$ left $\Lambda$ module if the trace ideal coincides with $\Lambda$ itself, or equivalently, if the trace ideal contains the identity element 1 , or what comes to the same, if there exist a finite number of $\Lambda$-homomorphisms $f_{i}: M \rightarrow \Lambda, i=1,2, \ldots, n$, and the same number of elements $a_{i} \in M, i=1,2, \ldots, n$, such that

$$
\sum_{i=1}^{n} f_{i}\left(a_{i}\right)=1
$$

The last condition is however equivalent with the condition that $\Lambda$ is, as a left A-module, a homomorphic image of the direct sum $M^{n}$ of $n$ copies of $M$, because the above equality implies that the mapping $\left(x_{1}, x_{2}, \ldots, x_{n}\right) \rightarrow \sum_{i=1}^{n} f_{i}\left(x_{i}\right), x_{i} \in M$, is an epimorphism of $M^{n}$ onto $\Lambda$ and, besides, every homomorphism $M^{n} \rightarrow \Lambda$ is given in the form $\left(x_{1}, x_{2}, \ldots, x_{n}\right) \rightarrow \sum_{i=1}^{n} f_{i}\left(x_{i}\right)$ with suitable homomorphisms $f_{i}$ : $M \rightarrow \Lambda$. Thus, $M$ is completely faithful if and only if $\Lambda$ is an homomorphic image of, or equivalently, since $\Lambda$ is projective, $\Lambda$ is a direct summand of a direct sum of copies of $M .^{*}$ Now, we shall call $M$ upper distinguished if every simple left $\Lambda$-module is a homomorphic image of $M$, while we call $M$ lower distinguished if $M$ contains an isomorphic image of every simple left $A$-module. A completely faithful module $M$ is necessarily upper distinguished. For, if $\mathfrak{l}$ is any maximal left ideal of $\Lambda$ there exists a homomorphism $f: M \rightarrow \Lambda$ such that $f(M)$ is not contained in $\mathfrak{l}$ and so the mapping $x \rightarrow f(x)+\mathfrak{l}$ gives an epimorphism of $M$ onto the simple module $\Lambda / \mathfrak{l}$. The converse is not true in general. But we have

TheоRem 1. Under the assumption that $M$ be projective, $M$ is completely faithful if and only if $M$ is upper distinguished.

For, suppose $M$ is not completely faithful, and let $\mathfrak{l}$ be a maximal left ideal containing the trace ideal of $M$. Then any homomorphism of $M$ into $A / \mathfrak{l}$ is, since $M$ is projective, factored into a product $h \circ f$ of the natural epimorphism

3) The notion was called generator by Bass [4] from the category theoretical view point, while it is to be noted that the notion is different from full faithfulness defined in Morita [10].

4) Bass [4, Lemma 1]. 
$h: \Lambda \rightarrow \Lambda / f$ and a homomorphism $f: M \rightarrow \Lambda$. But $f(M)$ is in the trace ideal and so is in $\mathfrak{l}$, which implies $h(f(M))=0$. This shows, since $A / \mathfrak{l}$ is a simple left $\Lambda$-module, that $M$ is not upper distinguished.

Now, Morita obtained in [9, Lemma 3.3] a fundamental theorem on completely faithful modules, which we shall restate here in a slightly modified form :

TheOREm 2. Let $M$ be a left $\Lambda$-module, and let $\Gamma$ be the A-endomorphism ring of $M$, considered as a right operator ring on $M$.

(i) If $M$ is completely faithful as A-module then $M$ is finitely generated and projective as $\Gamma$-module and $\Lambda$ coincides with the $\Gamma$-endomorphism ring of $M$.

(ii) If $M$ is finitely generated and projective as 1 -module then $M$ is completely faithful as $\Gamma$-module.

While (i) can be proved as in the second half of the proof of $[9$, Lemma 3.3], the same method is applicable to proving (ii). Namely, we associate with each $\varphi:{ }_{\Lambda} M \rightarrow{ }_{\Lambda} \Lambda$ a unique $f: M_{\Gamma} \rightarrow \Gamma_{\Gamma}$ such that $\varphi(x) y=x f(y)$ for all $x$, $y \in M$. The finite generatedness and the projectivity of ${ }_{\Lambda} M$ means the existence of a finite number of $\varphi_{i}:{ }_{\Lambda} M \rightarrow{ }_{\Lambda} \Lambda, i=1,2, \ldots, n$, and $a_{i} \in M, i=1,2, \ldots, n$, such that $x=\sum \varphi_{i}(x) a_{i}$ for all $x \in M$. Let $f_{i}: M_{\Gamma} \rightarrow \Gamma_{\Gamma}$ be the mappings corresponding to $\varphi_{i}$. Then we should have $x=\sum \varphi_{i}(x) a_{i}=\sum x f_{i}\left(a_{i}\right)=x \sum f_{i}\left(a_{i}\right)$ for all $x \in M$, which means that $\sum f_{i}\left(a_{i}\right)=1$, i.e., $M$ is completely faithful.

If we combine Theorem 1 with Theorem 2, we have the following theorem for projective modules:

Theorem 3. Let $\Lambda, \Gamma$ be two rings, and let $P$ be a two-sided $\Lambda$ - $\Gamma$-module. Then the following conditions are equivalent:

(1) $P$ is, as A-module, finitely generated, projective and upper distinguished, and $\Gamma$ coincides with the A-endomorphism ring of $M$.

(2) $P$ is, as $\Gamma$-module, finitely generated, projective and upper distinguished, and $A$ coincides with the $\Gamma$-endomorphism ring of $M$.

(3) The covariant functors $\operatorname{Hom}_{\Lambda}(P$,$) and P \otimes_{\Gamma}$ provide mutually inverse category-isomorphisms between the category of left A-modules and the category of left $\Gamma$-modules.

This theorem is regarded as a generalization of Morita [9, Theorem 7.3], and the equivalence of (1) (or of (2)) and (3) can be proved in the similar way 
as in the proof of the theorem.

It may be interesting to compare this theorem with the following theorem, which was proved in Morita [9, Theorem 6.3] or in Azumaya [3, Theorems 6, 8, 9]:

Theorem 4. Let $\Lambda, \Gamma$ be two rings, and let $Q$ be a two-sided $\Lambda$ - $\Gamma$-module. Then the following conditions are equivalent:

(1) $\Lambda$ is left-Artinian, $Q$ is, as A-module, finitely generated, injective and lower distinguished, and $\Gamma$ coincides with the A-endomorphism ring of $M$.

(2) $\Gamma$ is right-Artinian, $Q$ is, as $\Gamma$-module, finitely generated, injective and lower distinguished, and $A$ coincides with the $\Gamma$-endomorphism ring of $M$.

(3) The contravariant functors $\operatorname{Hom}_{\Lambda}(, Q)$ and $\operatorname{Hom}_{\Gamma}(, Q)$ provide mutually inverse dual category-isomorphisms between the category of finitely generated left $\Lambda$-modules and the category of finitely generated right $\Gamma$-modules.

It is to be noted that, while Theorem 4 is regarded as a dualization of Theorem 3, the assumptions of the chain conditions for $\Lambda$ and $\Gamma$ as well as the finite generatedness for modules are indispensable in Theorem 4, contrary to in Theorem 3.

Finally, we shall consider the case where $\Lambda$ is a subring of some over-ring:

THEOREM 5. Let $\Omega$ be a ring and $A$ its unital subring. ${ }^{51}$ Then $\Omega$ is, as a left $\Lambda$-module, completely faithful if and only if $A$ is a direct summand of $\Omega$.

Proof. The "if" part is evident. In order to prove the "only if" part, suppose $\Omega$ be completely faithful, i.e., there exist homomorphisms $f_{i}:{ }_{\Lambda} \Omega \rightarrow{ }_{\Lambda} \Lambda$ and elements $a_{t} \in \Omega, i=1,2, \ldots, n$, such that $\sum_{i=1}^{n} f_{i}\left(a_{i}\right)=1$. Consider then the mapping $x \rightarrow \sum_{i=1}^{n} f_{i}\left(x a_{i}\right), x \in \Omega$. This is obviously a homomorphism: ${ }_{\Lambda} \Omega \rightarrow{ }_{\Lambda} \Lambda$, and the above equality shows that the image of the identity 1 is also the identity 1 , which implies that ${ }_{\Lambda} \Omega$ is a direct sum of $\Lambda$ and the kernel of the homomorphism.

Corollary. Let $\Omega$ be a ring and $\Lambda$ its unital subring. If $\Omega$ is, as a left $\Lambda$ module, projective and upper distinguished, then $A$ is a direct summand of $\Omega$.

This follows immediately from Theorems 1 and 5 .

5) A subring is called unital if it contains the identity element of the over-ring. 
If we combine Theorem 5 with (the left-right analogy of) Theorem 2, (ii), we have Nakayama [13, Proposition 1]: Let $\Omega$ be a ring, and let $\Gamma$ be a ring of endomorphisms of the additive group $\Omega$ containing all right multiplications by elements of $\Omega$ and such that $\Omega$ is $\Gamma$-projective. Then if we put $\Lambda$ the $\Gamma$-endomorphism ring of $\Omega,{ }^{6)} \Lambda$ is a direct summand of $\Omega$ as 1 -modules.

2. If a left $\Lambda$-module $M$ is completely faithful then $M$ is faithful, that is, 0 is the only element of $\Lambda$ which annihilates $M$. Indeed, if $f_{i}:{ }_{\Lambda} M \rightarrow{ }_{\Lambda} \Lambda$ and $a_{i} \in M, i=1,2, \ldots, n$, satisfy the equality $1=\sum_{i=1}^{n} f_{i}\left(a_{i}\right)$ and if $\lambda \in \Lambda$ is an annihilator of $M$ then $\lambda=\lambda \sum_{i=1}^{n} f_{i}\left(a_{i}\right)=\sum_{i=1}^{n} \lambda f_{i}\left(a_{i}\right)=\sum_{i=1}^{n} f_{i}\left(\lambda a_{i}\right)=0$. The converse is not necessarily true. However, the following theorem gives a characterization of those rings $A$ for which the converse is true:

Theorem 6. Let $\Lambda$ be a ring. In order that every faithful left 1 -module be completely faithful it is necessary and sufficient that $\Lambda$ be left self-injective and a finite direct sum of indecomposable left ideals each of which contains a minimal left ideal of $\Lambda$.

Proof. We first prove the necessity: Let $A$ be a simple left $\Lambda$-module and let $Q(A)$ denote the injective envelope of $A$ in the sense of Eckmann-Schopf [5]. Then $Q(A)$ is an essential extension of $A$, or equivalently, every nonzero $A$-submodule of $Q(A)$ contains $A$ (and hence $A$ is the only minimal $A$ submodule of $Q(A)$ ). We let $A$ range over (up to isomorphism) all simple left $A$-modules and consider the direct sum $Q$ of all corresponding injective envelopes $Q(A)$ :

$$
Q=\sum_{A} \oplus Q(A)
$$

Then $Q$ is faithful. For, let $\mu$ be any non-zero element of $\Lambda$. Then the left annihilator $l(\mu)$ of $\mu$ in $\Lambda$ is a left ideal different from $\Lambda$. Therefore, there exists a maximal left ideal $\mathfrak{l}$ of $\Lambda$ containing $l(\mu)$. The factor module $\Lambda / \mathfrak{l}$ is a simple left $A$-module and so is isomorphic to some $A$, which we shall denote by $A_{0}$. Then $A_{0}$ must contain a non-zero element $a$ such that $\mathrm{\Upsilon} a=0$. Since $\lambda \mu=0$ implies $\lambda \in l(\mu) \subseteq \mathfrak{l}$ whence $\lambda a=0$, the mapping $\lambda \mu \rightarrow \lambda a, \lambda \in \Lambda$, is

6) $\Lambda$ is regarded as a unital subring of $\Omega$, and in fact $\Lambda$ is characterized as the subring of those elements $\lambda$ of $\Omega$ for which $\lambda \gamma=\lambda(1 \gamma)$ for all $\gamma \in \Gamma$, where 1 means the identity element of $\Omega$ and so $1 \gamma$ is an element of $\Omega$. 
well-defined and is a $\Lambda$-homomorphism of $\Lambda \mu$ into $A_{0}$. Since $Q\left(A_{0}\right)$ is an injective module containing $A_{0}$, this homomorphism can be extended to a $\Lambda$-homomorphism of $\Lambda$ into $Q\left(A_{0}\right)$, or what is the same, there exists an element $q$ of $Q\left(A_{0}\right)$ such that $\mu q=a$. Thus we have $\mu Q\left(A_{0}\right) \neq 0$ whence $\mu Q \neq 0$, showing that $Q$ is faithful. $Q$ is therefore completely faithful according to our assumption. This means that $\Lambda$ is, for some positive integer $n$, a homomorphic image of the direct $\operatorname{sum} Q^{n}=\sum_{A} \oplus Q(A)^{n}$ of $n$ copies of $Q$. Let $x$ be an element of $Q$ whose image is the identity element 1 . Then $x$ is in some partial finite direct sum, say, $Q^{\prime}=Q\left(A_{1}\right)^{n} \oplus Q\left(A_{2}\right)^{n} \oplus \cdots \oplus Q\left(A_{r}\right)^{n}$, and consequently $\Lambda$ is also a homomorphic image of, or equivalently (since $\Lambda$ is projective), a direct summand of $Q^{\prime}$. Since each $Q\left(A_{i}\right)$ is injective, their finite direct sum $Q^{\prime}$ is injective and hence its direct summand $\Lambda$ is also injective, i.e., $\Lambda$ is left self-injective. On the other hand, since each $Q\left(A_{i}\right)$ is an essential extension of $A_{i}$, their direct sum $Q^{\prime}$ is an essential extension of $A^{\prime}=A_{1}^{n} \oplus A_{2}^{n} \oplus \cdots \oplus A_{r}^{n}$. Therefore, $\Lambda$ is, as a $\Lambda$-submodule of $Q^{\prime}$, an essential extension of $\Lambda \cap A^{\prime}$. Thus $Q^{\prime}$ and $\Lambda$ are the injective envelopes of $A^{\prime}$ and $\Lambda \cap A^{\prime}$ respectively. Now $A \cap A^{\prime}$ is, as a $A$-submodule of the completely reducible module $A^{\prime}=A_{1}^{n} \oplus A_{2}^{n} \oplus \cdots \oplus A_{r}^{n}$, also completely reducible and is isomorphic to a finite sum of the form $A_{1}^{g(1)}$ $\oplus A_{2}^{g(2)} \oplus \cdots \oplus A_{r}^{g(r)}$, where each multiplicity $g(i)$ of $A_{i}$ is an integer such that $0 \leq g(i) \leq n$. Then $\Lambda$ is, because of the uniqueness of injective envelope, isomorphic to the injective envelope $Q\left(A_{1}\right)^{g(1)} \oplus Q\left(A_{2}\right)^{g(2)} \oplus \cdots \oplus Q\left(A_{r}\right)^{g(r)}$ of $A_{1}^{g(1)} \oplus A_{2}^{g(2)} \oplus \cdots \oplus A_{r}^{g(\gamma)}$. This, together with that each $Q\left(A_{i}\right)$ is, since every non-zero its $\Lambda$-submodule contains $A_{i}$, indecomposable, implies that $\Lambda$ is decomposed into a direct sum of indecomposable left ideals each of which contains a (unique) minimal left ideals of $\Lambda$.

Next, we prove the sufficiency: Let $\Lambda$ be a self-injective ring and let

$$
\Lambda=\mathfrak{l}_{1} \oplus \mathfrak{l}_{2} \oplus \cdots \oplus \mathfrak{l}_{s}
$$

be a decomposition of $\Lambda$ into a finite direct sum of indecomposable left ideals $\mathfrak{l}_{i}$ which have minimal left subideals $m_{i}$. Then each $\mathfrak{l}_{i}$ is injective and therefore contains an injective envelope of $m_{i}$. The injective envelope is a direct summand of and hence, since $\mathfrak{r}_{i}$ is indecomposable, coincides with $\mathfrak{r}_{l}$. In particular, it follows that $\mathfrak{l}_{i}$ and $\mathfrak{r}_{j}$ are isomorphic if and only if $m_{i}$ and $m_{j}$ are isomorphic. Let us assume, without loss of generality, that among the $s$ left ideals $\mathfrak{l}_{1}, \mathfrak{l}_{2}$, 
$\ldots, \mathfrak{l}_{s}$, the first $k$ ideals $\mathfrak{l}_{1}, \mathfrak{l}_{2}, \ldots, \mathfrak{l}_{k}$ are pair-wise non-isomorphic and such that any other $\mathfrak{l}_{i}, k<i \leq s$, is isomorphic to one of these. For each index $i$ between 1 and $k$, we denote by $g(i)$ the number of those indices $j$ between 1 and $s$ such that $\mathfrak{r}_{j}$ is isomorphic to $\mathfrak{l}_{i}$ : thus

$$
\Lambda \cong \mathfrak{I}_{1}^{g(1)} \oplus \mathrm{I}_{2}^{g(2)} \oplus \cdots \oplus \mathbb{I}_{k}^{g(k)}
$$

Let now $M$ be a faithful left $\Lambda$-module. Consider any $\mathfrak{l}_{i}$ with $1 \leq i \leq k$. Since the minimal left subideal $\mathfrak{m}_{i}$ of $\mathfrak{l}_{i}$ is non-zero, there exists an element $a_{i}$ in $M$ such that $\mathfrak{m}_{i} a_{i} \neq 0$. We then consider the homomorphism $\lambda \rightarrow \lambda a_{i}, \lambda \in \mathfrak{r}_{i}$, of $\mathfrak{I}_{i}$ into $M$. The kernel of this homomorphism cannot contain $m_{i}$ and so must be 0 , since $r_{i}$ is an essential extension of $m_{i}$. Thus the homomorphism is a monomorphism, so that the image $\mathfrak{l}_{i} a_{i}$ is a $\Lambda$-submodule of $M$ isomorphic to $\mathfrak{l}_{i}$ and hence is the injective envelope of its unique minimal $\Lambda$-submodule $m_{i} a_{i}$. We shall next prove that the sum of these $k$ submodules $\mathfrak{r}_{1} a_{1}, \mathfrak{r}_{2} a_{2}, \ldots, \mathfrak{r}_{k} a_{k}$ is direct. ${ }^{7)}$ For the purpose, suppose for some $j(\leq k)$ the sum $\mathfrak{l}_{1} a_{1}+\mathfrak{I}_{2} a_{2}+\cdots+$ $\mathfrak{l}_{j} a_{j}$ be direct, and consider the intersection $\left(\mathfrak{l}_{1} a_{1} \oplus \mathfrak{l}_{2} a_{2} \oplus \cdots \oplus \mathfrak{r}_{j} a_{j}\right) \cap \mathfrak{r}_{j+1} a_{j+1}$. If this was non-zero, then this would, since $r_{j+1} a_{j+1}$ is an essential extension of $\mathrm{m}_{j+1} a_{j+1}$, contain $\mathrm{m}_{j+1} a_{j+1}$; thus $\mathrm{m}_{j+1} a_{j+1}$ would be contained in $\mathfrak{r}_{1} a_{1} \oplus \mathrm{I}_{2} a_{2} \oplus \cdots$ $\oplus \mathfrak{r}_{j} a_{j}$ and therefore, for some $i(\leq j)$, the $i$-th component of $\mathrm{m}_{j+1} a_{j+1}$ (with respect to this direct decomposition) would be non-zero. Since $m_{j+1} a_{j+1}$ is a simple $\Lambda$-module isomorphic to $m_{j+1}$, the non-zero component is isomorphic to $\mathrm{m}_{j+1}$ too, while the component must contain whence coincide with the unique submodule $m_{i} a_{i}$ of $l_{i} a_{i}$. This is a contradiction, because $m_{j+1}$ and $m_{i}$ are not isomorphic. Thus $\left(\mathfrak{l}_{1} a_{1} \oplus \mathfrak{l}_{2} a_{2} \oplus \cdots \oplus \mathfrak{l}_{j} a_{j}\right) \cap \mathfrak{l}_{j+1} a_{j+1}=0$, and this proves our assertion by induction on $j$. Now, the direct sum $\mathfrak{r}_{1} a_{1} \oplus \mathfrak{r}_{2} a_{2} \oplus \cdots \oplus \mathfrak{l}_{k} a_{k}$ is injective and so is a direct summand of $M$. Put $n=\max (g(1), g(2), \ldots, g(k))$. Then $\Lambda \cong \mathfrak{l}_{1}^{g(1)} \oplus \mathfrak{l}_{2}^{g(2)} \oplus \cdots \oplus \mathfrak{I}_{k}^{g(k)} \cong\left(\mathfrak{l}_{1} a_{1}\right)^{g(1)} \oplus\left(\mathfrak{l}_{2} a_{2}\right)^{g(2)} \oplus \cdots \oplus\left(\mathfrak{l}_{k} a_{k}\right)^{g(k)}$ is a direct summand of $\left(\mathfrak{l}_{1} a_{1}\right)^{n} \oplus\left(\mathfrak{l}_{2} a_{2}\right)^{n} \oplus \cdots \oplus\left(\mathfrak{l}_{k} a_{k}\right)^{n} \cong\left(\mathfrak{l}_{1} a_{1} \oplus \mathfrak{I}_{2} a_{2} \oplus \cdots \oplus \mathfrak{I}_{k} a_{k}\right)^{n}$ and thus is a direct summand of $M^{n}$, which shows that $M$ is completely faithful.

Now, the following theorem gives us a detailed information of the structure of rings characterized in the preceding theorem:

Theorem 7. Let $\Lambda$ be a ring characterized in Theorem 6 . Then (1) the

7) The proof given below for this fact is similar to that of Jans [8, Theorem 3.2]. 
residue class ring $\Lambda / J$ modulo the Jacobson radical $J$ of $\Lambda$ is a semi-simple ring with minimum condition, (2) $A$ is left as well as right lower distinguished, (3) $\Lambda$ is a finite direct sum of indecomposable right ideals each of which is an essential extension of a minimal right ideal of $\Lambda^{8)}$ (4) the left socle and the right socle of $\Lambda$ coincide.

Proof. Let $\Lambda=\mathfrak{I}_{1} \oplus \mathfrak{I}_{2} \oplus \cdots \oplus \mathfrak{I}_{s}$ be a decomposition of $\Lambda$ into a finite direct sum of indecomposable left ideals $\mathfrak{l}_{i}$ having minimal left subideals $\mathfrak{m}_{i}$, as in the proof of the preceding theorem, and let $1=e_{1}+e_{2}+\cdots+e_{s}, e_{i} \in \mathrm{t}_{i}$, be the decomposition of the identity 1 relative to this direct decomposition. Then $e_{1}$, $e_{2}, \ldots, e_{s}$ are pair-wise orthogonal primitive idempotent elements, and each $e_{i}$ generates $\mathfrak{l}_{i}: \Lambda e_{i}=\mathfrak{l}_{i}$. Since $\mathfrak{l}_{i}$ is an injective envelope of $\mathrm{m}_{i}$, the endomorphism ring of $\mathfrak{l}_{i}$ is a local ring, i.e., the sum of any two non-automorphisms of $\mathfrak{l}_{i}$ is also non-automorphism, because non-automorphisms are in this case characterized as those endomorphisms which annihilate $m_{i}$. According to Azumaya $[2$, Theorem $1,(\mathrm{i})]$, there is, for any non-zero idempotent element $f$ of $\Lambda$, at least one $\mathfrak{l}_{i}$ such that $\mathfrak{l}_{i} f$ is isomorphic to $\mathfrak{l}_{i}$ and is an indecomposable direct summand left ideal of $\Lambda$. This implies, since $\mathfrak{I}_{i} f$ is contained in $\Lambda f$, there exists a primitive idempotent element $e$ such that $\Lambda e=\mathfrak{l}_{i} f$ and $e$ is contained in $f: f e f=e$. But since $\mathfrak{l}_{i} f \cong \mathfrak{l}_{i}$, the endomorphism ring $e \Lambda e$ of $\Lambda e=\mathfrak{l}_{i} f$ is isomorphic to that of $\mathfrak{l}_{i}$ and hence is a local ring. It follows from these that the set $C$ of those elements $c$ of $\Lambda$ for which $\Lambda c$ (or equivalently, $c \Lambda$ ) contains no non-zero idempotent element forms a two-sided ideal of $\Lambda^{9)}$ The Jacobson radical $J$ is contained in $C$, since $J$ does not contain any non-zero idempotent element. On the other hand, if we apply [2, Theorem 2, (ii)] to the finite direct decomposition of $\Lambda$, we can conclude that every element $\lambda$ of $\Lambda$ such that $\lambda \equiv 1(\bmod C)$ is an inversible element, or what comes to the same, every element of $C$ is quasi-regular. Thus $C$ is contained in and so is equal to $J: C$ $=J$. Let now $e$ be a primitive idempotent element of $\Lambda$. Let $\bar{e}$ denote the residue class of $e$ modulo $J$. Then the left ideal $\bar{\Lambda} \bar{e}$ of the residue class ring $\bar{\Lambda}=\Lambda / J$ is simple. For, let $\bar{\lambda}$ be any non-zero element of $\bar{\Lambda} \bar{e}$, and take a representative $\lambda$ of the class $\bar{\lambda}$ from $\Lambda e$. Then $\lambda$ is not in $J$, so that $\Lambda \lambda$ contains a non-zero idempotent element $f$. Since $\Lambda f \subseteq \Lambda \lambda \subseteq \Lambda e, \Lambda f$ is a non-zero

8) It is an open question whether $\Lambda$ is right self-injective or not.

9) See Azumaya [2, p. 117]. 
direct summand of $\Lambda e$, which implies, since $\Lambda e$ is indecomposable, that $\Lambda f=\Lambda e$. Thus $\Lambda \lambda=\Lambda e$ whence $\bar{\Lambda} \bar{\lambda}=\bar{\Lambda} \bar{e}$, showing the simplicity of $\bar{\Lambda} \bar{e}$. In particular, each $\bar{\Lambda} \bar{e}_{i}$ is a simple left ideal of $\bar{\Lambda}$, while $\bar{e}_{1}, \bar{e}_{2}, \ldots, \bar{e}_{s}$ are pair-wise orthogonal idempotent elements of $\bar{\Lambda}$ whose sum is the identity $\overline{1}: \bar{e}_{1}+\bar{e}_{2}+\cdots+\bar{e}_{s}=\overline{1}$. Thus $\bar{\Lambda}$ is the direct sum of simple left ideals $\bar{\Lambda} \bar{e}_{1}, \bar{\Lambda} \bar{e}_{2}, \ldots, \bar{\Lambda} \bar{e}_{s}$, and $\bar{\Lambda}$ is a semi-simple ring with minimum condition: $\bar{\Lambda}=\bar{\Lambda} \bar{e}_{1} \oplus \bar{\Lambda} \bar{e}_{2} \oplus \cdots \oplus \bar{\Lambda} \bar{e}_{s}$. Let $k$ and $g(i), i=1,2, \ldots, k$, have the same meanings as in the proof of Theorem 6. Namely, let $\mathfrak{l}_{1}=\Lambda e_{1}, \mathfrak{l}_{2}=\Lambda e_{2}, \ldots, \mathfrak{l}_{k}=\Lambda e_{k}$ are pair-wise non-isomorphic, and let $\Lambda \cong\left(\Lambda e_{1}\right)^{g(1)} \oplus\left(\Lambda e_{2}\right)^{g(2)} \oplus \cdots \oplus\left(\Lambda e_{k}\right)^{g(k)}$. Then, by taking modulo $J$, we have $\bar{\Lambda} \equiv\left(\bar{\Lambda} \bar{e}_{1}\right)^{g(1)} \oplus\left(\bar{\Lambda} \bar{e}_{2}\right)^{g(2)} \oplus \cdots \oplus\left(\bar{\Lambda} \bar{e}_{k}\right)^{g(k)}$. This implies that any simple left $\Lambda$-module is isomorphic to at least one of $\bar{\Lambda} \bar{e}_{i}$ 's $(i \leq k)$. On the other hand, the (unique) minimal left subideals $\mathrm{m}_{1}, \mathrm{~m}_{2}, \ldots, \mathrm{m}_{k}$ of $\Lambda e_{1}, \Lambda e_{2}, \ldots, \Lambda e_{k}$ are pairwise non-isomorphic (since each $\Lambda e_{i}$ is an injective envelope of $m_{i}$ ). Thus, it follows that the $k$ simple left $\Lambda$-modules $\bar{\Lambda} \bar{e}_{1}, \bar{\Lambda} \bar{e}_{2}, \ldots, \bar{\Lambda} \bar{e}_{s}$ are pairwise nonisomorphic and there exists a permutation $\pi=(\pi(1), \pi(2), \ldots, \pi(k))$ of $(1$, $2, \ldots, k$ ) such that $\mathrm{m}_{i} \cong \bar{\Lambda} \bar{e}_{\pi(i)}$ for each $i$, which means that $\Lambda$ is left lower distinguished.

The remaining part of our theorem can be proved more or less in the same way as in Azumaya [3, Theorem 3]. Namely, let $\mu$ be a non-zero element of $\Lambda$. Then its left annihilator $l(\mu)$ is a proper left ideal and hence there exists a maximal left ideal $\mathfrak{l}$ which contains $l(\mu)$. The right annihilator $r(\mathfrak{l})$ of $\mathfrak{l}$ is a right ideal. We take any element $\nu$ of $r(l)$. Then the mapping $\lambda \mu \rightarrow$ $\lambda \nu, \lambda \in \Lambda$, is well-defined, and this is a $\Lambda$-homomorphism: $\Lambda \mu \rightarrow \Lambda$. Since $\Lambda$ is left self-injective, there should exists an element $\lambda^{\prime}$ of $\Lambda$ such that $\mu \lambda^{\prime}=\nu$. Thus we have $\mu \Lambda \supseteq r(\mathfrak{l})$. On the other hand, if $\mathfrak{l}$ is any maximal left ideal then $\Lambda$ contains, since it is left lower distinguished, an isomorphic image of the simple left $\Lambda$-module $\Lambda / \mathfrak{l}$, which means that $r(\mathfrak{l}) \neq 0$. If moreover we take $\mu$ any non-zero element of $r(l)$ in this case then $\mathfrak{l}$ (is contained in whence) coincides with $l(\mu)$ and therefore $\mu \Lambda=r(\mathfrak{l})$. These facts together imply that the right annihilator $r(\mathfrak{l})$ of any maximal left ideal $\mathfrak{l}$ is a simple right ideal, conversely any simple right ideal is expressed as the right annihilator $r(\mathfrak{l})$ of some maximal left ideal $\mathfrak{l}$, and besides any non-zero right ideal of $A$ contains a simple right ideal. The right socle of $A$ is therefore equal to the sum of all those $r(1)$ 's. However, this is originally the left socle, because if $\mu(\neq 0)$ is any element of 
$r(l)$ then $\Lambda \mu$ is a simple left ideal isomorphic to $\Lambda / \mathfrak{l}$, while if $\mathrm{m}$ is a simple left ideal and $\mu(\neq 0)$ any element of $m$ then the left annihilator 1 of $\mu$ is, since $\Lambda / \mathfrak{l}$ is isomorphic to $\Lambda \mu=\mathfrak{m}$, a maximal left ideal and $\mu$ is in $r(\mathfrak{l})$. Thus the right socle and the left socle coincide, which we shall denote by $S$. On the other hand, since $\Lambda / J$ is a semi-simple ring with minimum condition, the right annihilator $r(J)$ of the radical $J$ (not only contains but also) coincides with the (left) socle $S$, because $r(J)$ is regarded as a left $\Lambda / J$-module and so is completely reducible: $r(J)=S$. (Similarly, the left annihilator $l(J)$ coincides with $S$.) Now, consider a primitive idempotent $e_{i}, 1 \leq i \leq k$. Since $\bar{\Lambda} \bar{e}_{i} \oplus \bar{\Lambda}$ $\left(\overline{1}-\bar{e}_{i}\right)=\bar{\Lambda}$, it follows that $\bar{\Lambda} \bar{e}_{i} \cong \bar{\Lambda} / \bar{\Lambda}\left(\overline{1}-\bar{e}_{i}\right) \cong \Lambda /\left(\Lambda\left(1-e_{i}\right)+J\right)$, which shows that $\Lambda\left(1-e_{i}\right)+J$ is a maximal left ideal of $\Lambda$. Therefore its right annihilator $r\left(\Lambda\left(1-e_{i}\right)+J\right)=r\left(1-e_{i}\right) \cap r(J)=e_{i} \Lambda \cap S=e_{i} S$ must be a simple right ideal. This means, since $S$ is the right socle of $\Lambda$, that $e_{i} \Lambda \cap S=e_{i} S$ is a unique minimal right subideal of $e_{i} \Lambda$ and hence every non-zero right subideal of $e_{i} \Lambda$ contains $e_{i} S$, that is, $e_{i} \Lambda$ is an essential extension of $e_{i} S$. Consider, on the other hand, the left ideal $\Lambda e_{i}$. Since it has a unique minimal left subideal $\mathrm{m}_{i}$ and since $S$ is the left socle of $\Lambda$, it follows necessarily $m_{i}=\Lambda e_{i} \cap S=S e_{i}$. Now there was a permutation $\pi$ of $(1,2, \ldots, k)$ such that $m_{i}=S e_{i} \cong \bar{\Lambda} e_{\pi(i)}$ for each $i$. Hence it follows $e_{\pi(i)} S e_{i} \neq 0$. But this also implies that the simple right ideal $e_{\pi(i)} S$ is isomorphic to the simple right $\Lambda$-module $\bar{e}_{i} \bar{\Lambda}: e_{\pi(i)} S \cong \bar{e}_{i} \bar{\Lambda}$. Since this is the case for all $i=1,2, \ldots, k$, it follows that $A$ is right lower distinguished. If we notice further that the decomposition $1=e_{1}+e_{2}+\cdots+e_{s}$ into orthogonal primitive idempotent elements $e_{i}$ yields the decomposition $\Lambda=e_{1} \Lambda \oplus e_{2} \Lambda \oplus \cdots$ $\oplus e_{s} \Lambda$ into a direct sum of indecomposable right ideals $e_{i} \Lambda$, we have thus completed the proof of our theorem.

Remark. Y. Utumi has obtained independently the following theorem which is virtually the same as our Theorem 6: In order that every faithful left $A$ module be completely faithful it is necessary and sufficient that (i) $A$ be left self-injective, (ii) $\Lambda / J$ be a semi-simple ring with minimum condition, and (iii) every non-zero left ideal contain a minimal left ideal. His method of the proof is different from ours, and the writer hopes that he will publish it somewhere.

\section{REFERENCES}

[1] M. Auslander and O. Goldman, Maximal orders, Trans. Amer. Math. Soc., 97 (1960), pp. 1-24. 
[2] G. Azumaya, Corrections and supplements to my paper concerning Krull-RemakSchmidt's theorem, Nagoya Math. J., 1 (1950), pp. 117-124.

[3] G. Azumaya, A duality theory for injective modules, Amer. J. Math., 81 (1959), pp. 249-278.

[4] H. Bass, The Morita theorems, Mimeographed note.

[5] B. Eckmann and A. Schopf, Über injective Moduln, Arch. Math., 4 (1953), pp. 75-78.

[6] S. Eilenberg and T. Nakayama, On the dimension of modules and algebras II, Nagoya Math. J., 9 (1955), pp. 1-16.

[ 7 ] M. Ikeda, A characterization of quasi-Frobenius rings, Osaka Math. J., 4 (1952), pp. 203-210.

[8] J. P. Jans, Projective injective modules, Pacific J. Math. 9 (1959), pp. 1103-1108.

[9] K. Morita, Duality for modules and its applications to the theory of rings with minimum condition, Sci. Rep. Tokyo Kyoiku Daigaku, 6 (1958), pp. 83-142.

[10] K. Morita, Category-isomorphisms and endomorphism rings of modules, Trans. Amer. Math. Soc., 103 (1962), pp. 451-469.

[11] T. Nakayama, On Frobeniusean algebras I, Ann. of Math., 40 (1939), pp. 611-633.

[12] T. Nakayama, On Frobeniusean algebras II, Ann. of Math., 42 (1941), pp. 1-21.

[13] T. Nakayama, On a generalized notion of Galois extensions of a ring, Osaka Math. J., 15 (1963), pp. 11-23.

[14] C. J. Nesbitt and R. M. Thrall, Some ring theorems with applications to modular representations, Ann. of Math., 47 (1946), pp. 551-567.

Added in proof. After submitting this paper, the writer has found that our Theorem 5 was already obtained by B. Müller in Hilfssatz 1 of the first of the following papers :

Quasi-Frobenius-Erweiterungen, Math. Z. 85 (1964), pp. 345-368,

Quasi-Frobenius-Erweiterungen II, Math. Z. 88 (1965), pp. 380-409.

In this connection, the writer would like to note that Hilfssatz 24 of the second paper (actually, Auslander-Buchsbaum-Goldman's theorem) can also be proved by combining our Theorem 1 with the following theorem:

THeorem 8. Let $\Lambda$ be commutative and $M$ a finitely generated, faithful $\Lambda$-module. Then $M$ is upper distinguished.

Proof. Let $u_{1}, u_{2}, \ldots, u_{n}$ be generators of $M$. Let $p$ be a maximal ideal of $\Lambda$. Then $p M \neq M$. For, if $p M=M$ then there exist, for each $i=1,2, \ldots, n$, elements $a_{i j} \in \mathfrak{p}$ such that $u_{i}=\sum_{j=1}^{n} a_{i j} u_{j}$. This implies that the characteristic polynomial $\operatorname{det}\left(\delta_{i j}-a_{i j}\right)$ of the $n \times n$ matrix $\left(a_{i j}\right)$ annihilates each $u_{i}$ whence $M$. Since $M$ is faithful it follows $\operatorname{det}\left(\delta_{i j}-a_{i j}\right)=0$, while since every $a_{i j}$ is in $\mathfrak{p}$ it follows $\operatorname{det}\left(\delta_{i j}-a_{i j}\right) \equiv \operatorname{det} \delta_{i j}=1(\bmod \mathfrak{p})$, which contradicts to the assump- 
tion that $1 \notin \mathfrak{p}$. Thus $\mathfrak{p} M \neq M$, and so $M / \mathfrak{p} M$ is regarded as a non-zero vector space over the residue class field $\Lambda / \mathfrak{p} . \Lambda / p$ is therefore a homomorphic image of $M / p M$ whence of $M$.

University of Massachusetts and Indiana University 